\title{
Chikungunya Virus-associated Long-term Arthralgia: A 36-month Prospective Longitudinal Study
}

\author{
Clémentine Schilte $^{1,29}$, Frédérik Staikovsky ${ }^{39}$, Thérèse Couderc ${ }^{4,59}$, Yoann Madec ${ }^{6}$, \\ Florence Carpentier ${ }^{7,8}$, Somar Kassab ${ }^{3}$, Matthew L. Albert ${ }^{1,2,9}$, Marc Lecuit ${ }^{4,5,9,10^{\top}} *$, Alain \\ Michault ${ }^{119_{*}}$
}

1 Unité Immunobiologie des Cellules Dendritiques, Department of Immunology, Institut Pasteur, Paris, France, 2 Institut National de la Santé et de la Recherche Médicale (INSERM) U818, Paris, France, 3 Emergency Department, Pôle des Spécialités de l'Urgence, Centre Hospitalier Régional de La Réunion, Groupe Hospitalier Sud Réunion, Saint Pierre, La Réunion, France, 4 Biology of Infection Unit, Institut Pasteur, Paris, France, 5 INSERM U1117, Paris, France, 6 Unité d'Epidémiologie des maladies émergentes, Institut Pasteur, Paris, France, 7 AgroParisTech, Ecologie Adaptation et Interaction, Paris, France, 8 INRA, UR1290, Thiverval-Grignon, France, 9 Université Paris Descartes, Sorbonne Paris Cité, Paris, France, 10 Division of Infectious Diseases, Hôpital Necker Enfants malades, Paris, France, 11 Department of Microbiology, Pôle de biologie, Centre Hospitalier Régional de La Réunion, Groupe Hospitalier Sud Réunion, Saint Pierre, La Réunion, France

\begin{abstract}
Background: Arthritogenic alphaviruses, including Chikungunya virus (CHIKV), are responsible for acute fever and arthralgia, but can also lead to chronic symptoms. In 2006, a Chikungunya outbreak occurred in La Réunion Island, during which we constituted a prospective cohort of viremic patients $(n=180)$ and defined the clinical and biological features of acute infection. Individuals were followed as part of a longitudinal study to investigate in details the long-term outcome of Chikungunya.

Methodology/Principal Findings: Patients were submitted to clinical investigations 4, 6, 14 and 36 months after presentation with acute CHIKV infection. At 36 months, 22 patients with arthralgia and 20 patients without arthralgia were randomly selected from the cohort and consented for blood sampling. During the 3 years following acute infection, $60 \%$ of patients had experienced symptoms of arthralgia, with most reporting episodic relapse and recovery periods. Long-term arthralgias were typically polyarthralgia $(70 \%)$, that were usually symmetrical $(90 \%)$ and highly incapacitating $(77 \%)$. They were often associated with local swelling (63\%), asthenia (77\%) or depression (56\%). The age over 35 years and the presence of arthralgia 4 months after the disease onset are risk factors of long-term arthralgia. Patients with long-term arthralgia did not display biological markers typically found in autoimmune or rheumatoid diseases. These data helped define the features of CHIKV-associated chronic arthralgia and permitted an estimation of the economic burden associated with arthralgia.
\end{abstract}

Conclusions/Significance: This study demonstrates that chronic arthralgia is a frequent complication of acute Chikungunya disease and suggests that it results from a local rather than systemic inflammation.

\footnotetext{
Citation: Schilte C, Staikovsky F, Couderc T, Madec Y, Carpentier F, et al. (2013) Chikungunya Virus-associated Long-term Arthralgia: A 36-month Prospective Longitudinal Study. PLoS Negl Trop Dis 7(3): e2137. doi:10.1371/journal.pntd.0002137

Editor: Sunit Kumar Singh, Centre for Cellular and Molecular Biology, India

Received September 11, 2012; Accepted February 11, 2013; Published March 21, 2013

Copyright: (c) 2013 Schilte et al. This is an open-access article distributed under the terms of the Creative Commons Attribution License, which permits unrestricted use, distribution, and reproduction in any medium, provided the original author and source are credited.

Funding: This study was funded by the CRVOI (Centre de Recherche et de Veille sur les maladies émergentes dans I'Océan Indien), Institut Pasteur, Inserm (Institut National de la Santé et de la Recherche Médicale), Labex IBEID, Fondation pour la Recherche Médicale, BNP-Paribas Foundation and Mairie de Paris. The funders had no role in study design, data collection and analysis, decision to publish, or preparation of the manuscript.

Competing Interests: The authors have declared that no competing interests exist.

*E-mail: marc.lecuit@pasteur.fr (ML); alain.michault@chr-reunion.fr (AM)

9 These authors contributed equally to this work.

- ML and AM also contributed equally to this work.
}

\section{Introduction}

Chikungunya virus (CHIKV) is an arthropod-borne virus that belongs to the Alphavirus genus. Chikungunya disease is characterized by polyarthralgia, sometimes associated with rash. The articular symptoms, often debilitating, usually resolve within weeks, but have been reported to last for months, even though the natural history of this infection has not been precisely studied in prospective studies $[1,2,3]$.

In 2005, CHIKV emerged in islands of Indian Ocean including La Réunion, a French overseas department, and approximately one third of the inhabitants (i.e. $\sim 300,000$ ) was infected at the end of the outbreak in $2006[4,5]$. Compared to earlier outbreaks, this episode occurred in a highly medicalized area. Moreover previously unreported severe forms of Chikungunya were observed, such as encephalopathy [6,7], and mother-to-child CHIKV transmission was demonstrated, leading to severe neonatal CHIKV infection [5]. In the wake of this outbreak, CHIKV also re-emerged in India with over 1 million cases $[8,9]$. In 2007, CHIKV emerged for the first time in Europe, causing an outbreak in Italy [10].

We have described the clinical and biological features of acute CHIKV infection in a prospective cohort of patients with positive blood CHIKV RT-PGR [11]. It included all patients referred to the Emergency Department in Saint-Pierre de la Réunion with 


\section{Author Summary}

Chikungunya virus (CHIKV) is transmitted to human by mosquitoes. It is a re-emerging virus that has a risk to spread globally, given the expanding dissemination of its mosquito vectors. Chikungunya disease is characterized by acute transient febrile arthralgic illness, but can also lead to chronic incapacitating arthralgia. We have conducted a prospective longitudinal study to investigate in details long-term outcome of CHIKV infection. We found that $60 \%$ of patients experienced arthralgia 36 months after the onset of acute disease. Arthralgia affected most often multiple sites and were usually incapacitating. In addition to arthralgia, many patients suffered from myalgia and cutaneous lesions and several cognitive dysfunctions. We also showed that age over 35 years and the presence of arthralgia 4 months after the onset of disease are risk factors for long-term arthralgia. Patients with long-term arthralgia did not display biological markers typically found in autoimmune or rheumatoid diseases. This study demonstrates that chronic arthralgia is a frequent complication of acute Chikungunya disease and suggests that it results from a local rather than systemic inflammation.

febrile arthralgia between March and May 2006. As little is known about long-term outcome of CHIKV infection, we conducted a prospective longitudinal study to describe in details the specific clinical and biological features of chronic arthralgia, as well as clinical signs associated to this pathology. We evaluated the consequences of long-term arthralgia on patients' daily and social life, looked for risk factors associated with them and estimated their economic impact.

\section{Materials and Methods}

\section{Ethics \& STROBE statement}

Ethical clearance was obtained from the «Comité de Protection des Personnes Sud-Ouest et Outre-Mer III» of Saint-Pierre, La Réunion, Paris (CCP 2008/65, n 2008-A00999-46). CHIKIMMUNOPATH received approval from the ethical committee for studies with human subjects (CPP) of Bordeaux and the National Commission for Informatics and Liberty (CNIL). Written informed consent was obtained from patients included in the CHIK-IMMUNOPATH study. The study respects the STROBE statement (supporting information S1).

\section{Patients}

We studied a cohort of patients $(n=180)$ enrolled for febrile arthralgia to the Emergency Department of the Groupe Hospitalier Sud Réunion between March 2005 and May 2006 [11]. Patients were interviewed by telephone 4, 6, 14 and 36 months (M4, M6, M14 and M36) after the viremic phase, using the same questionnaire as that used at day 0 (D0) (supporting information S2). At M36 after the acute phase, all patients who agreed to participate to a complementary study (CHIK-IMMUNOPATH) and who were arthralgic were interviewed and underwent clinical examination. Among them, 22 patients with arthralgia (ART+) and 20 patients without persisting arthralgia (ART - ) were randomly selected from the cohort. They signed a written consent and a blood sample was collected.

\section{Laboratory tests performed at M36}

Blood cell count was performed and viremia was tested by qRTPCR [12]. Both serum anti-CHIKV IgG and IgM specific antibodies were screened. An enzyme-linked immunosorbent assay (ELISA) was performed with CHIKV antigen [12]. The avidity of anti-CHIKV IgG was tested by ELISA in the presence or absence of urea $8 \mathrm{M}$ [13]. Geometric Mean Antibody Titer (GMAT) was calculated as previously described [14].

Plasmatic protein electrophoresis was performed and C-reactive protein (CRP) concentration was measured. The presence of antinuclear, anti-dsDNA, anti-endomysium autoantibodies, anti-cyclic citrullinated peptide antibody (ACCP) and cryoglobulinemia were investigated. Samples were transported at $37^{\circ} \mathrm{C}$ to research cryoglobulins. Sera were sent to Myriad RBM (Austin,Texas) and analyzed by Luminex using the inflammation MAP. Assays are run according to CLIA guidelines and in all cases, $>100$ beads per analyte were measured with $\mathrm{CV}<10 \%$ for values that are above the limit of quantification for the given assay.

\section{Statistical analysis}

For each time point, the proportion of patients with monoarthralgia (1 site), oligoarthralgia (2-3 sites) or polyarthralgia (4 sites or more) were compared using a Chi-2 test.

A logistic regression model was used to identify factors associated with long-term arthralgia, defined as presence of arthralgia at M14. We studied factors at D0, including demographic factors (gender and age), biological markers, hospitalization and comorbidities, and factors measured at M4 (arthralgia, treatment, and quality of life). All factors associated with long-term arthralgia with a $\mathrm{p}$-value $<0.15$ in univariate analysis were entered in the multivariate model. A step-by-step backward procedure was then used to identify factors significantly associated with long-term arthralgia. A sensitivity analysis was also conducted, following the same procedure, defining long-term persistence of arthralgia as the presence of arthralgia at M36. To address the lost of follow up at M36, we looked for parameters that differentiate the patients who were lost between M14 and M36 and these who were followed.

Statistical analyses were performed using the STATA software (Stata Corporation, College Station, Texas, USA); all significance tests were two-sided and $\mathrm{p}$-values $<0.05$ were considered significant. Luminex data were mined using the Omniwiz software (Biowisdom) and Mann-Whitney analysis is reported. False discovery rate (FDR or $q$-values) were calculated as correction for multiple analyte testing.

To characterize the spatiotemporal evolution of arthralgia, we considered different types of arthralgia. For a given site at a given time point, arthralgia was defined either as a "persistent symptom" if the affected site was the same as that reported during the previous time point, as "a relapse of an the acute symptom" if the site affected was the same as that at the acute phase, or as "a new symptom" if this site was not affected at the acute phase, or as a "migrating symptom" if the arthralgia was localized at a site distinct from that previously reported. For a given patient, these categories were not mutually exclusive. For modeling migratory arthralgia, we divided joints into three groups: upper limb joints (hand, wrist, elbow), mid body joints, (shoulder, spine, hip) and lower limb joints (foot, ankle, knee), and considered two migratory probabilities for migration to sites within to the same group (e.g., hand to wrist) or to a different group (e.g., hand to foot) (Supporting information S3 and Table S1).

\section{Results}

Characteristics of long-term arthralgia in CHIKV-infected patients

At M4, M6, M14 and M36 after their inclusion as acute CHIKV-infected patients, all patients were interviewed using a questionnaire to monitor persistence of arthralgia, other clinical signs and treatments. The number of patients that participated is 


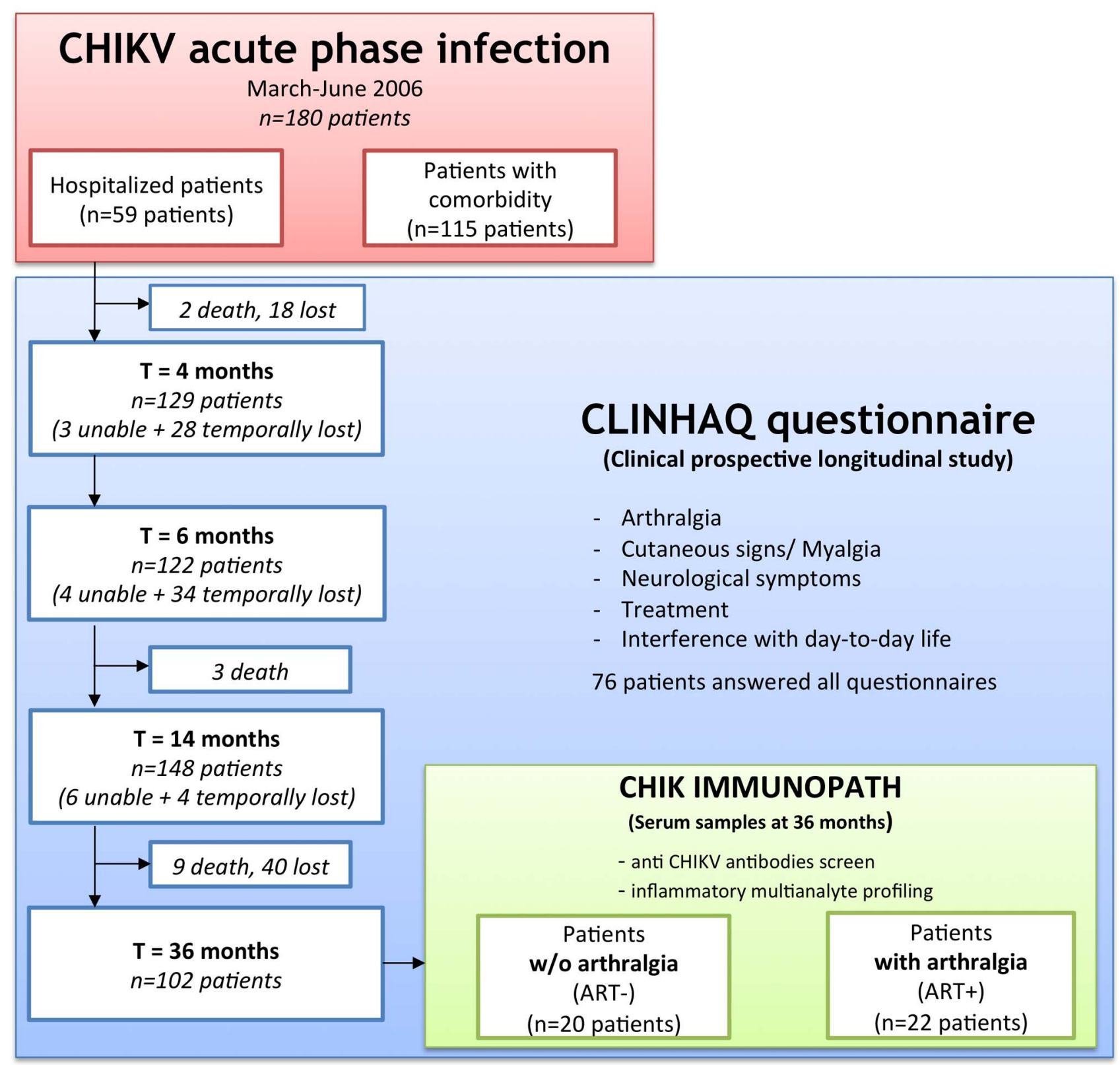

Figure 1. Diagram of the clinical study. "unable": patients who were reached but unable to answer to the questionnaire, "lost": patient lost of follow up until the end of the study, "temporally lost": not reached at a specific timepoint, "death": dead patients.

doi:10.1371/journal.pntd.0002137.g001

provided in Figure 1. There is an important lost of follow up between M14 and M36 however we did not identify a bias associated with it. Among the 180 patients, 76 patients were followed at all time points of the study.

The percentage of patients suffering from long-term arthralgia decreased after CHIKV acute infection and stabilized around $60 \%$ (Figure 2A). Of note, all patients suffered from arthralgia at D0. Among them, only 5 on $180(2.8 \%)$ suffered from joint pain prior to CHIKV infection.

Most patients had intermittent arthralgia, with recovery and relapse. For each time point, 25 to $40 \%$ of patients complained of permanent arthralgia. Among the 76 patients that could be followed at each time point, $45 \%$ had arthralgia at all time, $24 \%$ experienced partial recovery at M4, M6 or M14 then relapses, and $31 \%$ fully recovered from acute symptoms. Among patients who experienced chronic symptoms at M36, 43.5\% reported arthralgia triggered by a change in ambient temperature, $8 \%$ by physical effort. At M36, arthralgia caused stiffness in $75.5 \%$ of patients with symptoms, and $67.7 \%$ of the patients reported a need of morning stretching (time of 32 minutes, standard deviation (SD) 37 minutes, range 5-180 minutes).

We monitored arthralgia in 9 anatomical sites (Figure 2B). Arthralgia in upper limbs mostly affected fingers and wrists, while lower limbs arthralgia mostly affected knees and ankles. At each time point, these locations remain significantly the most affected (Mac Nemar test for matched pairs of subjects). Importantly, arthralgia were typically symmetrical $(90 \%)$.

We then investigated whether the number of arthralgic sites diminished in patients still suffering from arthralgia among the 76 patients followed at all time points. The number of arthralgia sites 

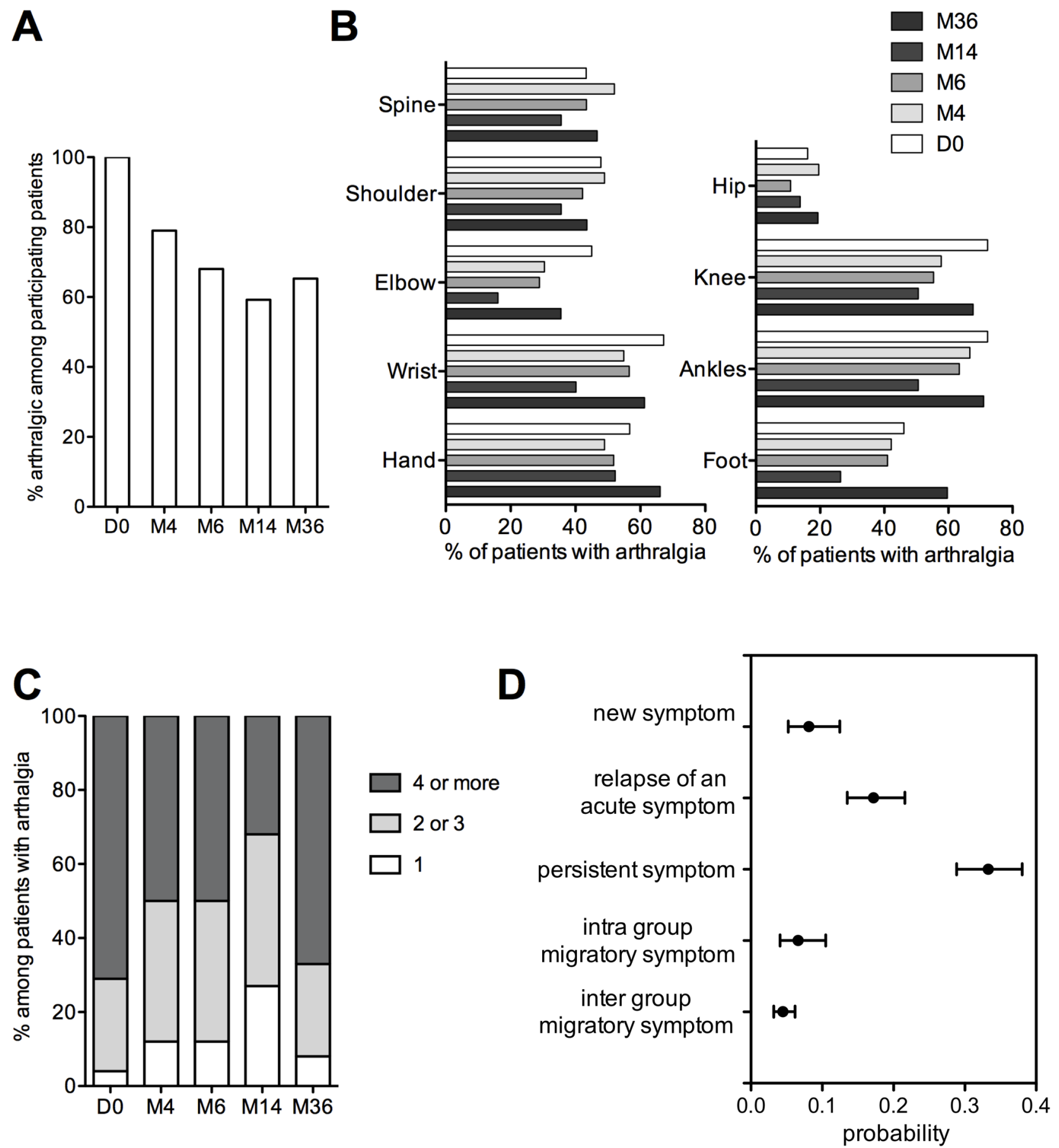

Figure 2. Evolution and characterization of arthralgia in CHIKV patients. (A) Percentage of patients with arthralgia among participating patients, (B) localization of arthralgia among patients with arthralgia, (C) number of arthralgia sites among the 76 patients answering at every time point and having arthralgia, (D) complete model presenting the probability for one joint to be painful depending on its previous state. doi:10.1371/journal.pntd.0002137.g002

decreased until M14, with only 30\% of patients suffering from polyarthralgia (number of arthralgia sites $>2$ ) (Figure 2C). Despite an increase of arthralgic sites at M36, there is an overall significant decrease of the number of painful joints during the study period $(\mathrm{p}<0.01)$.

We attempted to model the spatiotemporal evolution of arthralgia, as defined in the Materials and Methods section. We found that "persistent" symptoms had the strongest effect, as its probability of occurrence was three times higher than a "new" symptom (Figure 2D). The probability of relapse of an "acute" symptom was twice the appearance of a "new" symptom. Finally, "migratory" symptoms tended to be intra-group as compared to inter-group migrations.

Other clinical signs associated with CHIKV chronic disease and their consequences

Patients with arthralgia at M36 showed other clinical symptoms, including local swelling, cutaneous symptoms, myalgia and osteoligamentaous pain (Table 1). Local swelling localized to affected joints for $63 \%$ of patients. Moreover, sleep, memory or 
Table 1. Clinical signs and treatment in arthralgic and non-arthralgic patients 36 months after the acute phase.

\begin{tabular}{|c|c|c|c|}
\hline & Patients with arthralgia $(n=62)$ & Patients w/o arthralgia $(n=33)$ & Chi-2 test ${ }^{\S}$ \\
\hline \multicolumn{4}{|l|}{ Clinical signs } \\
\hline Local swelling & $39(62.9 \%)$ & $0(0 \%)$ & $* * *$ \\
\hline Osteo-ligamentous pain & $22(35.5 \%)$ & $0(0 \%)$ & *** \\
\hline Myalgia & $24(38.7 \%)$ & $0(0 \%)$ & $* * *$ \\
\hline Cutaneous lesion & $31(50.0 \%)$ & $5(15.1 \%)$ & $* * *$ \\
\hline Asthenia & $48(77.4 \%)$ & $7(21.2 \%)$ & $* * *$ \\
\hline Sleeping disorder & $35(56.4 \%)$ & $3(9.1 \%)$ & $* * *$ \\
\hline Dysgeusia & $11(17.7 \%)$ & $3(9.1 \%)$ & NS \\
\hline Depression & $31(50.0 \%)$ & $2(6 \%)$ & $* * *$ \\
\hline Memory disorder & $27(43.5 \%)$ & $2(6 \%)$ & $* * *$ \\
\hline Concentration disorder & $24(38.7 \%)$ & $2(6 \%)$ & $* * *$ \\
\hline \multicolumn{4}{|l|}{ Treatment } \\
\hline Followed by a general practioner & $50(80.6 \%)$ & $1(3 \%)$ & *** \\
\hline Treatment & $51(82.2 \%)$ & $0(0 \%)$ & *** \\
\hline Continous treatment & $25(49 \%)$ & $0(0 \%)$ & *** \\
\hline Paracetamol & $45(72.5 \%)$ & $0(0 \%)$ & $* * *$ \\
\hline Morphinic & $0(0 \%)$ & $0(0 \%)$ & \\
\hline Non-steroidal anti-inflammatory drugs & $12(23.5 \%)$ & $0(0 \%)$ & ** \\
\hline Corticosteroids & $3(5.9 \%)$ & $0(0 \%)$ & NS \\
\hline
\end{tabular}

${ }^{\text {s } n s: ~ N o n ~ s i g n i f i c a n t . ~}$

${ }^{* *} \mathrm{p}<0.01$.

$* * * \mathrm{p}<0.001$

doi:10.1371/journal.pntd.0002137.t001

Table 2. Impact of arthralgia on daily life for patients at M36.

\begin{tabular}{|c|c|c|c|}
\hline & Arthralgic patients $(n=62)$ & Non arthralagic patients $(n=33)$ & Chi-2 test $^{\mathrm{s}}$ \\
\hline \multicolumn{4}{|l|}{ Impact on quality of life } \\
\hline rising from a chair & $39(48.4 \%)$ & $0(0 \%)$ & $* * *$ \\
\hline walking & $34(54.8 \%)$ & $0(0 \%)$ & $* * *$ \\
\hline picking up an object & $34(54.8 \%)$ & $0(0 \%)$ & $* * *$ \\
\hline opening a bottle & $33(53.2 \%)$ & $0(0 \%)$ & $* * *$ \\
\hline drinking glass & $26(41.9 \%)$ & $0(0 \%)$ & $* * *$ \\
\hline to wash oneself & $23(37.1 \%)$ & $0(0 \%)$ & $* * *$ \\
\hline At least one of these disabilities & $48(77.4 \%)$ & $0(0 \%)$ & $* * *$ \\
\hline \multicolumn{4}{|l|}{ Impact on working life } \\
\hline With activity & $15(24.2 \%)$ & $17(51.5 \%)$ & \\
\hline No impact & $3(20 \%)$ & $16(94.1 \%)$ & $* * *$ \\
\hline Physical impact & $12(80 \%)$ & $1(5.9 \%)$ & \\
\hline low impact & $7(46.7 \%)$ & $1(5.9 \%)$ & \\
\hline moderate impact & $3(20 \%)$ & $0(0 \%)$ & \\
\hline high impact & $2(13.3 \%)$ & $0(0 \%)$ & \\
\hline \multicolumn{4}{|l|}{ Impact on leisure-time } \\
\hline No impact & $13(39.4 \%)$ & $31(93.9 \%)$ & \\
\hline Physical impact & 49 (79\%) & $2(6.1 \%)$ & $* * *$ \\
\hline low impact & $8(24.2 \%)$ & $1(3 \%)$ & \\
\hline moderate impact & $18(54.5 \%)$ & $0(0 \%)$ & \\
\hline high impact & $23(69.7 \%)$ & $1(3 \%)$ & \\
\hline
\end{tabular}

$s_{* *} p<0.01 .{ }^{* * *} p<0.001$.

doi:10.1371/journal.pntd.0002137.t002 
Table 3. Identification of risk factors for persistence of arthralgia 14 months after CHIKV acute disease (univariate analysis).

\begin{tabular}{|c|c|c|c|c|}
\hline & Patients at M14 N & $\begin{array}{l}\text { Patients with arthralgia } \mathrm{N} \\
(\%)\end{array}$ & Odd Ratio $\left(95 \% \mathrm{Cl}^{5}\right)$ & $\mathbf{p}$ \\
\hline \multicolumn{5}{|l|}{ Gender } \\
\hline Male & 73 & $41(56.2 \%)$ & $0.78(0.40-1.51)$ & 0.46 \\
\hline Female & 74 & $46(62.2 \%)$ & 1 & \\
\hline \multicolumn{5}{|l|}{ Age (years) } \\
\hline$\leq 35$ & 30 & $9(30.0 \%)$ & $0.18(0.05-0.57)$ & 0.005 \\
\hline $36-50$ & 28 & $19(67.9 \%)$ & $0.87(0.26-2.84)$ & \\
\hline $51-60$ & 24 & $17(70.8 \%)$ & 1 & \\
\hline $61-70$ & 20 & $15(75.0 \%)$ & $1.24(0.32-4.73)$ & \\
\hline$>70$ & 45 & 27 (60.0\%) & $0.62(0.21-1.79)$ & \\
\hline \multicolumn{5}{|l|}{ Hospitalization at D0 } \\
\hline No & 101 & $61(60.4)$ & 1 & 0.66 \\
\hline Yes & 46 & $26(56.5)$ & $0.85(0.42-1.73)$ & \\
\hline \multicolumn{5}{|c|}{$\begin{array}{l}\text { Viral quantification at DO (copies/ } \\
\mathrm{mL} \text { ) }\end{array}$} \\
\hline$\leq 100.000$ & 70 & $39(55.7)$ & 1 & 0.41 \\
\hline$>100.000$ & 77 & $48(62.3)$ & $1.32(0.68-2.54)$ & \\
\hline \multicolumn{5}{|l|}{ CRP at D0 (unit) } \\
\hline$<10$ & 20 & $7(35.0)$ & 1 & 0.02 \\
\hline$>10$ & 126 & $79(62.7)$ & $3.12(1.16-8.38)$ & \\
\hline Missing & 1 & $1(100.0)$ & No estimable & \\
\hline \multicolumn{5}{|l|}{ Diabetes at D0 } \\
\hline No & 109 & $58(53.2)$ & 1 & 0.01 \\
\hline Yes & 38 & $29(76.3)$ & $2.83(1.23-6.54)$ & \\
\hline \multicolumn{5}{|l|}{ Arthralgia at M4 } \\
\hline No & 26 & $5(19.2)$ & $0.10(0.04-0.30)$ & $<0.001$ \\
\hline Yes & 99 & $69(60.7)$ & 1 & \\
\hline Missing & 22 & $13(59.1)$ & $0.63(0.24-1.62)$ & \\
\hline \multicolumn{5}{|l|}{ Arthralgia at M4 } \\
\hline No & 26 & $5(19.2)$ & $0.25(0.08-0.78)$ & $<0.001$ \\
\hline Yes. intermittent & 43 & $21(48.8)$ & 1 & \\
\hline Yes. permanent & 56 & $48(85.7)$ & $6.28(2.41-16.38)$ & \\
\hline Missing & 22 & $13(59.1)$ & $1.51(0.53-4.28)$ & \\
\hline \multicolumn{5}{|c|}{ Memory disorder at M4 } \\
\hline No & 87 & $41(47.1)$ & 1 & $<0.001$ \\
\hline Yes & 38 & $33(86.8)$ & $7.40(2.64-20.75)$ & \\
\hline Missing & 22 & $13(59.1)$ & $1.62(0.63-4.18)$ & \\
\hline \multicolumn{5}{|c|}{ Concentration disorder at M4 } \\
\hline No & 94 & $45(47.9)$ & 1 & $<0.001$ \\
\hline Yes & 31 & 29 (93.6) & 15.79 (3.56-69.97) & \\
\hline Missing & 22 & $13(59.1)$ & $1.57(0.61-4.03)$ & \\
\hline
\end{tabular}

${ }^{\S}$ confidence interval.

doi:10.1371/journal.pntd.0002137.t003

concentration disorders and asthenia or depression are significantly associated with arthralgic patients.

The proportion of patients with arthralgia who attended a physician or received a treatment significantly increased between M4 and M36 ( $\mathrm{p}=0.01$ ) (data not shown), and reached $80 \%$ (Table 1). Similarly, the number of patients receiving a treatment increased and these treatments are statistically associated with the arthralgic status of the patient $(\mathrm{p}<0.001)$.
Arthralgia in patients at M36 were highly incapacitating for daily life tasks, professional life and spare-time activities (Table 2).

\section{Identification of risk factors for developing long-term CHIKV-associated arthralgia}

To identify risk factors associated with long-term arthralgia, we performed univariate and multivariate statistical analyses at M14, as the participation was higher than at M36 (Table 3). Gender was 
Table 4. Analytes titrated in arthralgic and non arthralgic patients 36 months after the acute phase.

\begin{tabular}{|c|c|c|c|c|c|c|c|c|c|c|c|c|}
\hline \multirow[b]{2}{*}{ ATTRIBUTE } & \multirow[b]{2}{*}{ Units } & \multirow[b]{2}{*}{ LOD } & \multicolumn{4}{|c|}{ Arthalgia ( $n=20$ patients) } & \multicolumn{4}{|c|}{ No Arthalgia ( $n=22$ patients) } & \multicolumn{2}{|l|}{ Statistic } \\
\hline & & & DET & $25 \%$ & MED & $75 \%$ & DET & $25 \%$ & MED & $75 \%$ & M-W PV & M-W FDR \\
\hline IP-10 & $\mathrm{pg} / \mathrm{mL}$ & 116 & 18 & 170 & 214 & 376 & 20 & 126 & 207 & 379 & 0.537 & 0.606 \\
\hline Alpha-2-Macroglobulin A2Macro & $\mathrm{mg} / \mathrm{mL}$ & 0.472 & 18 & 0.6 & 0.7 & 0.9 & 21 & 0.7 & 0.8 & 1.1 & 0.194 & 0.434 \\
\hline Alpha-1-Antitrypsin AAT & $\mathrm{mg} / \mathrm{mL}$ & 1.33 & 20 & 1.9 & 2.1 & 2.6 & 22 & 1.8 & 2.0 & 2.1 & 0.305 & 0.606 \\
\hline Beta-2-Microglobulin B2M & $\mathrm{ug} / \mathrm{mL}$ & 0.914 & 20 & 1.1 & 1.4 & 1.9 & 22 & 1.2 & 1.3 & 1.6 & 0.481 & 0.606 \\
\hline $\begin{array}{l}\text { Brain-Derived Neurotrophic Factor } \\
\text { BDNF }\end{array}$ & $\mathrm{ng} / \mathrm{mL}$ & 4.7 & 20 & 12 & 17 & 24 & 22 & 14 & 18 & 21 & 0.940 & 0.966 \\
\hline Complement C3 C3 & $\mathrm{mg} / \mathrm{mL}$ & 0.93 & 20 & 1.4 & 1.5 & 1.8 & 22 & 1.0 & 1.2 & 1.5 & 0.029 & 0.056 \\
\hline C-Reactive Protein CRP & $\mathrm{ug} / \mathrm{mL}$ & 0.063 & 20 & 1.5 & 2.8 & 5.8 & 22 & 0.5 & 1.3 & 2.4 & 0.035 & 0.056 \\
\hline Eotaxin-1 & $\mathrm{pg} / \mathrm{mL}$ & 70.8 & 20 & 165 & 195 & 290 & 22 & 154 & 238 & 329 & 0.705 & 0.920 \\
\hline Factor VII & $\mathrm{ng} / \mathrm{mL}$ & 142 & 20 & 443 & 529 & 632 & 22 & 324 & 383 & 561 & 0.020 & 0.056 \\
\hline Fibrinogen & $\mathrm{ug} / \mathrm{mL}$ & 0.93 & 20 & 1.6 & 1.9 & 2.4 & 22 & 1.4 & 2.0 & 2.4 & 0.950 & 0.966 \\
\hline Ferritin FRTN & $\mathrm{ng} / \mathrm{mL}$ & 14.7 & 20 & 34 & 112 & 156 & 22 & 68 & 159 & 273 & 0.034 & 0.056 \\
\hline $\begin{array}{l}\text { Granulocyte-Macrophage Colony- } \\
\text { Stimulating Factor }\end{array}$ & $\mathrm{pg} / \mathrm{mL}$ & 0 & 0 & 0.0 & 0.0 & 0.0 & 0 & 0.0 & 0.0 & 0.0 & $N A$ & $N A$ \\
\hline Haptoglobin & $\mathrm{mg} / \mathrm{mL}$ & 0.007 & 20 & 1.0 & 1.6 & 2.1 & 21 & 0.6 & 0.9 & 1.8 & 0.194 & 0.434 \\
\hline $\begin{array}{l}\text { Intercellular Adhesion Molecule } 1 \\
\text { ICAM-1 }\end{array}$ & $\mathrm{ng} / \mathrm{mL}$ & 32.1 & 20 & 88 & 98 & 119 & 22 & 87 & 107 & 121 & 0.801 & 0.920 \\
\hline Interferon gamma IFN-gamma & $\mathrm{pg} / \mathrm{mL}$ & 0.9 & 6 & 0.9 & 0.9 & 0.9 & 2 & 0.9 & 0.9 & 1.0 & 0.038 & 0.056 \\
\hline Interleukin-1 alpha IL-1 alpha & $\mathrm{pg} / \mathrm{mL}$ & 1.51 & 2 & 1.4 & 1.4 & 3.1 & 10 & 1.4 & 1.4 & 1.4 & 0.007 & 0.010 \\
\hline Interleukin-1 beta IL-1 beta & $p g / m L$ & 2.68 & 0 & 2.4 & 2.4 & 2.4 & 2 & 2.4 & 2.4 & 2.4 & 0.091 & 0.112 \\
\hline Interleukin-10 IL-10 & $\mathrm{pg} / \mathrm{mL}$ & 1.55 & 17 & 1.6 & 2.2 & 2.8 & 18 & 1.6 & 2.2 & 2.6 & 0.808 & 0.920 \\
\hline Interleukin-12 Subunit p40 IL-12p40 & $n g / m L$ & 0 & 0 & 0.0 & 0.0 & 0.0 & 0 & 0.0 & 0.0 & 0.0 & $N A$ & NA \\
\hline Interleukin-12 Subunit p70 IL-12p70 & $\mathrm{pg} / \mathrm{mL}$ & 29.3 & 0 & 29 & 29 & 29 & 3 & 29 & 29 & 29 & 0.051 & 0.056 \\
\hline Interleukin-15 IL-15 & $n g / m L$ & 0.2 & 5 & 0.2 & 0.2 & 0.4 & 11 & 0.2 & 0.2 & 0.2 & 0.021 & 0.056 \\
\hline Interleukin-17 IL-17 & $\mathrm{pg} / \mathrm{mL}$ & 0.9 & 9 & 0.9 & 0.9 & 1.5 & 11 & 0.9 & 0.9 & 1.5 & 0.838 & 0.920 \\
\hline Interleukin-18 IL-18 & $\mathrm{pg} / \mathrm{mL}$ & 61.3 & 20 & 117 & 135 & 235 & 22 & 130 & 153 & 189 & 0.811 & 0.920 \\
\hline $\begin{array}{l}\text { Interleukin-1 receptor antagonist } \\
\text { IL-1ra }\end{array}$ & $\mathrm{pg} / \mathrm{mL}$ & 50.2 & 15 & 87 & 113 & 154 & 22 & 49 & 87 & 129 & 0.090 & 0.112 \\
\hline Interleukin-2 IL-2 & $\mathrm{pg} / \mathrm{mL}$ & 2.8 & 0 & 2.8 & 2.8 & 2.8 & 2 & 2.8 & 2.8 & 2.8 & 0.573 & 0.606 \\
\hline Interleukin-23 IL-23 & $n g / m L$ & 0.4 & 8 & 0.4 & 0.4 & 0.7 & 8 & 0.4 & 0.4 & 0.7 & 0.787 & 0.920 \\
\hline Interleukin-3 IL-3 & $\mathrm{pg} / \mathrm{mL}$ & 5.1 & 1 & 5.1 & 5.1 & 5.1 & 0.0 & 5.1 & 5.1 & 5.1 & $N A$ & $N A$ \\
\hline Interleukin-4 IL-4 & $\mathrm{pg} / \mathrm{mL}$ & 2.6 & 1 & 2.6 & 2.6 & 2.6 & 5 & 2.6 & 2.6 & 2.6 & 0.111 & 0.112 \\
\hline Interleukin-5 IL-5 & $\mathrm{pg} / \mathrm{mL}$ & 3.8 & 11 & 3.8 & 4.8 & 6.8 & 16 & 3.4 & 3.8 & 5.1 & 0.168 & 0.406 \\
\hline Interleukin-6 IL-6 & $\mathrm{pg} / \mathrm{mL}$ & 5.8 & 1 & 5.8 & 5.8 & 5.8 & 0 & 5.8 & 5.8 & 5.8 & 0.133 & 0.352 \\
\hline Interleukin-7 IL-7 & $\mathrm{pg} / \mathrm{mL}$ & 5 & 4 & 5.0 & 5.0 & 5.0 & 1 & 5.0 & 5.0 & 5.0 & 0.112 & 0.112 \\
\hline Interleukin-8 IL-8 & $\mathrm{pg} / \mathrm{mL}$ & 2.8 & 19 & 9 & 17 & 46 & 20 & 9 & 11 & 24 & 0.650 & 0.897 \\
\hline $\begin{array}{l}\text { Monocyte Chemotactic Protein } 1 \\
\text { MCP-1 }\end{array}$ & $\mathrm{pg} / \mathrm{mL}$ & 33.7 & 20 & 105 & 170 & 216 & 22 & 129 & 150 & 172 & 0.435 & 0.606 \\
\hline $\begin{array}{l}\text { Macrophage Inflammatory Protein-1 } \\
\text { alpha MIP-1 alpha }\end{array}$ & $\mathrm{pg} / \mathrm{mL}$ & 10.6 & 15 & 11 & 16 & 46 & 16 & 11 & 27 & 36 & 0.869 & 0.933 \\
\hline $\begin{array}{l}\text { Macrophage Inflammatory Protein-1 } \\
\text { beta MIP-1 beta }\end{array}$ & $\mathrm{pg} / \mathrm{mL}$ & 112 & 20 & 194 & 284 & 431 & 22 & 242 & 269 & 328 & 0.950 & 0.966 \\
\hline Matrix Metalloproteinase-2 MMP-2 & $n g / m L$ & 0 & 0 & 0.0 & 0.0 & 0.0 & 0 & 0.0 & 0.0 & 0.0 & $N A$ & $N A$ \\
\hline Matrix Metalloproteinase-3 MMP-3 & $\mathrm{ng} / \mathrm{mL}$ & 2.31 & 20 & 2.9 & 5.7 & 8.8 & 22 & 5.5 & 6.9 & 10.3 & 0.162 & 0.406 \\
\hline Matrix Metalloproteinase-9 MMP-9 & $\mathrm{ng} / \mathrm{mL}$ & 1.3 & 12 & 1.2 & 1.3 & 1.3 & 15 & 1.2 & 1.3 & 1.3 & 0.305 & 0.606 \\
\hline $\begin{array}{l}\text { T-Cell-Specific Protein RANTES } \\
\text { RANTES }\end{array}$ & $\mathrm{ng} / \mathrm{mL}$ & 3.81 & 20 & 7 & 16 & 21 & 22 & 9 & 17 & 23 & 0.345 & 0.606 \\
\hline Stem Cell Factor SCF & $\mathrm{pg} / \mathrm{mL}$ & 60.4 & 20 & 155 & 192 & 229 & 22 & 140 & 167 & 219 & 0.239 & 0.514 \\
\hline $\begin{array}{l}\text { Tissue Inhibitor of } \\
\text { Metalloproteinases } 1 \text { TIMP-1 }\end{array}$ & $\mathrm{ng} / \mathrm{mL}$ & 68.7 & 20 & 120 & 132 & 169 & 22 & 126 & 144 & 177 & 0.623 & 0.897 \\
\hline $\begin{array}{l}\text { Tumor Necrosis Factor alpha TNF- } \\
\text { alpha }\end{array}$ & $\mathrm{pg} / \mathrm{mL}$ & 1.33 & 20 & 2.0 & 2.2 & 2.7 & 21 & 1.8 & 2.2 & 2.7 & 0.643 & 0.897 \\
\hline
\end{tabular}


Table 4. Cont.

\begin{tabular}{|c|c|c|c|c|c|c|c|c|c|c|c|c|}
\hline \multirow[b]{2}{*}{ ATTRIBUTE } & \multirow[b]{2}{*}{ Units } & \multirow[b]{2}{*}{ LOD } & \multicolumn{4}{|c|}{ Arthalgia ( $n=20$ patients) } & \multicolumn{4}{|c|}{ No Arthalgia ( $n=22$ patients) } & \multicolumn{2}{|l|}{ Statistic } \\
\hline & & & DET & $25 \%$ & MED & $75 \%$ & DET & $25 \%$ & MED & $75 \%$ & M-W PV & M-W FDR \\
\hline $\begin{array}{l}\text { Tumor Necrosis Factor beta } \\
\text { TNF-beta }\end{array}$ & $\mathrm{pg} / \mathrm{mL}$ & 12.1 & 1 & 12 & 12 & 12 & 1 & 12.1 & 12.1 & 12.1 & 0.612 & 0.897 \\
\hline $\begin{array}{l}\text { Tumor Necrosis Factor } \\
\text { Receptor-Like } 2 \text { TNFR2 }\end{array}$ & $\mathrm{ng} / \mathrm{mL}$ & 2.51 & 20 & 3 & 4 & 8 & 22 & 3.3 & 4.0 & 5.2 & 0.364 & 0.606 \\
\hline $\begin{array}{l}\text { Vascular Cell Adhesion Molecule-1 } \\
\text { VCAM-1 }\end{array}$ & $\mathrm{ng} / \mathrm{mL}$ & 323 & 20 & 460 & 595 & 707 & 22 & 490 & 539 & 640 & 0.606 & 0.606 \\
\hline Vitamin D-Binding Protein VDBP & $\mathrm{ug} / \mathrm{mL}$ & 114 & 20 & 158 & 273 & 372 & 22 & 237 & 259 & 303 & 0.762 & 0.920 \\
\hline $\begin{array}{l}\text { Vascular Endothelial Growth } \\
\text { Factor VEGF }\end{array}$ & $\mathrm{pg} / \mathrm{mL}$ & 288 & 20 & 458 & 628 & 763 & 22 & 492 & 579 & 786 & 0.811 & 0.920 \\
\hline von Willebrand Factor vWF & $\mathrm{ug} / \mathrm{mL}$ & 13.7 & 20 & 21 & 28 & 35 & 22 & 20 & 26 & 34 & 0.762 & 0.920 \\
\hline
\end{tabular}

DET: number of patients with detectable value within the group

MED: $25 \%$; 75\%:median and interquartile.

LOD: lowest limit of detection.

M-W PV: Mann Whithney $P$ value.

M-W FDR Mann Whitney with False Discovery Rate Correction.

doi:10.1371/journal.pntd.0002137.t004

not associated with long-term arthralgia, age less than 35 years was protective. Risk of arthralgia was not associated with indicators of the disease severity during the acute phase (viral load, duration of hospitalization or number of sites of arthralgia at D0) [11], however it was weakly associated with $\mathrm{C}$-reactive protein (CRP) level at D0. Diabetes was the only comorbidity found to be a risk factor for long-term arthralgia. Interestingly, arthralgia at M14 was strongly associated with arthralgia at M4, and even more if arthralgia was permanent at M4. Memory and concentration disorders at M4 were also identified as risk factors for developing long-term arthralgia. Arthralgia, memory disorders and concentration disorders at M4 were the only risk factors significantly and independently associated with long-term arthralgia. When longterm arthralgia was assessed at M36, results were very similar.

\section{Arthralgia status, systemic inflammation and autoimmunity markers}

At M36, 22 patients with arthralgia (ART+) and 20 patients without arthralgia (ART-) were randomly selected from the cohort to participate to the CHIK IMMUNOPATH study. Its aim was to titrate anti-CHIKV antibodies and identify a serum inflammatory or autoimmune signature associated with the arthralgia phenotype.

All patients were negative for CHIKV RT-PCR, and exhibited anti-CHIKV IgGs in serum, while a minority $(9.5 \%)$ harbored measurable levels of anti-CHIKV IgM. The activity of CHIKV IgG (GMAT) was significantly higher in ART+ patients (30) than in ART - patients (20), but antibody avidity was comparable in both groups (mean \pm SD: $31,6 \pm 20,4$ in ART + patients and $33,7 \pm 19,8$ in ART - patients). Although lymphopenia is a defining feature of acute CHIKV disease [11], it was a rare finding at M36 (data not shown). Plasma protein levels measured by electrophoresis and CRP concentration were within normal ranges. However, CRP levels were significantly higher in the $\mathrm{ART}+$ group than in the ART - group (mean $\pm \mathrm{SD}$ : $3.35 \pm 3.00 \mathrm{mg} / \mathrm{ml}$ and $1.85 \pm 2.49 \mathrm{mg} / \mathrm{ml}$, respectively; $\mathrm{p}=0.04)$.

We used Luminex xMAP technology to assay analytes in the serum of patients. Most analytes were undetectable in both groups of patients (Table 4). Five inflammation markers were significantly elevated in ART+ patients: factor VII, C3 complement component,
IL1 $\alpha$, IL1 5 and CRP (Figure 3). Ferritin level was significantly lower in ART+ patients than in ART - patients. These markers did not allow for the identification of a subgroup within the ART+ group, nor did they correlate one with another. No autoimmune marker and no anti-DNA antibody in the serum of ART+ patients were detected, although anti-nuclear antibodies were detected at low level in four ART+ patients. Three patients had elevated antinuclear antibodies, one in the ART+ group and two in the ARTgroup.

As it has been reported that CHIKV could evolve into rheumatoid arthritis [15], we screened for cyclic citrullinated protein antibodies. We also assayed for cryoglobulinemia and antiendomysium IgA antibodies. All patient were found to be negative.

\section{Estimation of the economic impact of CHIKV long-term arthralgia}

We estimated the annual economic burden of long-term arthralgia by taking into account the cost of medical visits, therapeutic treatment and the cost for lost work time due to injury or pain (using the population of La Réunion Island as a reference) (Table S2). We found that arthralgia secondary to the CHIKV outbreak in La Réunion in 2005-06 has resulted so far in an estimated total cost of up to 34 millions euros per year. This corresponds to $250 €$ per year and per patient with long-term arthralgia. However, it should be noted that this sum might be overestimated due to the bias in our cohort selection, as our cohort is likely composed of the most severely affected patients who were referred to the hospital during the acute phase.

\section{Discussion}

Our study is the first prospective cohort study on CHIKV longterm arthralgia that is based on the follow-up of patients who presented with acute CHIKV infection as the inclusion criterion. This study is also the first to define the evolution of CHIKVinduced arthralgia, mapping the frequency and location of arthralgic sites during a three year time period. We have also investigated the impact of CHIKV-chronic arthralgia on daily life of patients, identified clinical signs associated with arthralgia, and analyze biologic markers. Moreover we have evaluated associated risk factors and estimated the economic burden of this disease. 

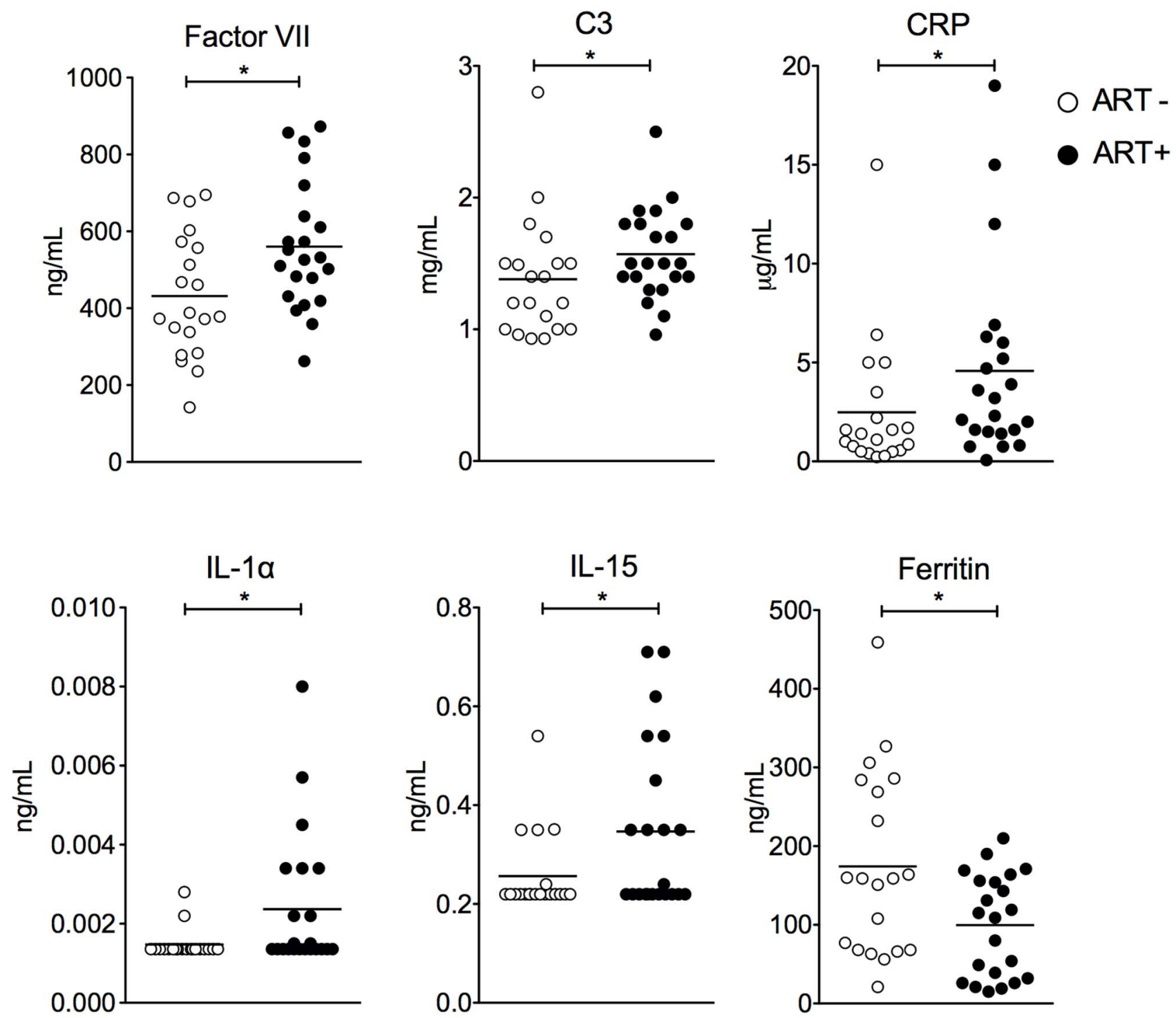

Figure 3. Distinctive markers in serum of patients with arthralgia (ART+ in black) versus patients without arthralgia (ART-, in white) at M36. SD: standard deviation, results of Mann Whitney test are shown. ${ }^{*} p<0.05, q=0.056$.

doi:10.1371/journal.pntd.0002137.g003

Together, these data allow us to define the features of CHIKVinduced chronic arthralgia (Table 5), as compared to other viral arthritis [16], and to establish a detailed understanding of the public health problem resulting from CHIKV-chronic arthralgia.

Our data reveal that more than $60 \%$ of CHIKV-infected patients suffer from arthralgia, 36 months after acute infection. This high percentage of patients with long-term symptoms was also reported by other studies of Italian cohorts and French cohorts of La Réunion Island or metropolitan France $[17,18,19,20,21]$ but is dramatically higher than documented in India and Senegal $[1,22,23,24,25]$. While this discrepancy may result from particular features of the CHIKV strain responsible for the La Réunion outbreak, data from Italy following the 2007 outbreak resulted from a CHIKV strain more closely related to the viral strain present in India [10], with more than $60 \%$ of CHIKV patients in Italy having reported myalgia, asthenia or arthralgia 12-13 months after the acute disease [20]. Alternatively, reported differences may be a result of different genetic backgrounds of these populations. As joint pain is considered a subjective symptom, it might also reflect a difference in pain threshold of patients or reporting from physicians, thus reflecting differences in health care practices.

Long-term CHIKV-associated arthralgia were mainly symmetrical, involving more than 2 different joints. Hand, wrist, ankle and knee were found to be the most affected, consistent with other studies [17,18]. Moreover, $60-80 \%$ of patients had relapsing arthralgia, while $20-40 \%$ had unremitting arthralgia. While some patients reported "migrating" arthralgia, most disease symptoms mapped to joints that were most painful during acute Chikungunya disease. Thus, symptoms at the chronic phase may be indirectly associated to virus replication at the time of acute infection $[26,27,28]$.

In addition to arthralgia, many patients suffered from myalgia and cutaneous lesions and several cognitive dysfunctions. Although study patients did not display neurological symptoms at the acute phase of disease, we cannot exclude that cognitive dysfunctions result from CHIKV spread in the GNS, as it has been reported that CHIKV disseminates to the GNS in humans and in animal 
Table 5. Features of patients with chronic CHIKV-associated arthralgia.

\begin{tabular}{ll}
\hline Age & $>35$ \\
Sex ratio (M/F) & $1 / 1$ \\
Number of sites & oligo or polyarthralgia \\
Sites & upper limbs : fingers. wrist \\
& lower limbs : knees. ankles \\
Type & symmetrical \\
& permanent or not \\
& migratory \\
& highly incapacitating \\
& morning stiffness duration average: \\
& $\approx 30$ minutes \\
General sign fever & no fever \\
Other clinical signs & edema \\
Risk factor & cutaneous lesion \\
\hline Laboratory tests & myalgia \\
& sleep and memory disorders \\
& CRP normal \\
& ACCP normal \\
& antinuclear normal \\
& anti CHIKV lgGs positive \\
& diabetes \\
\hline & \\
\hline & \\
&
\end{tabular}

models $[12,26,27,29]$. Similar to other studies, chronic arthralgia are considered incapacitating for daily life tasks and impacted professional activities and quality of life [20,21]. Beside this impact on patient, the economic burden of this long-term pathology is also very significant, independently of the cost of the acute disease [30].

The longitudinal design of our study enabled us to identify risk factors for development of long-term arthralgia. Individuals over the age of 35 years or with diabetes were more likely to suffer from chronic arthralgia. The age has been reported to be a risk factor with some cohorts $[21,31,32]$, but not in others [18,33]. None of our available parameters to measure the severity of acute disease were associated with long-term arthralgia. This may be explained by differences in the way to measure disease severity in other studies [18]. Importantly, we show that the presence and intensity of arthralgia at M4 after the onset of the acute disease is a good predictor of long-term arthralgia.

Our study did not identify positive markers for autoimmune or rheumatoid arthritis. Additionally, we failed to identify systemic biomarkers associated with the arthralgic phenotype. Nevertheless, a slightly more elevated inflammatory status is found in a subset of arthralgic patients who have detectable serum level of IL1 $\alpha$, IL15 and slight elevation in Factor VII, C3 and CRP. This signature differs from that observed at the onset of the infection, when circulating virus is detectable and type I interferon, IP10, MCP1, ISG15 are highly elevated [34,35,36]. Others have identified IL6 and GM-CSF or IL12 as being specifically associated with longterm arthralgia [32,33]. However, these studies were performed much earlier in the chronic phase (2-3 months and one year after disease onset).
Our study shows that anti-CHIKV antibody titers were more elevated in ART+ patients than in ART- patients. This is in agreement with a recent study [37]. This higher level of antibodies could be associated with a more severe acute infection [37]. However, in our study, the level of antibody at M36 did not correlate with acute disease severity. Alternatively, this could reflect a persisting antigenic stimulation in ART+ patients (see below). Interestingly, it has been reported that viremia level at the acute phase correlates with a faster appearance of neutralizing antibodies and a better recovery $2-3$ months after the acute phase [38].

Similarly to CHIKV, other so called "arthritogenic" alphaviruses, notably Ross River virus (RRV), are known to cause acute as well as chronic arthralgia [39]. Our data indicates that chronic symptoms are linked to the initial local joint inflammation and are not associated with markers of systemic inflammation or autoimmunity. A local inflammation of the joint could be maintained by the local persistence or delayed clearance of viral antigens. This is consistent with report of Hoarau et al. [32] who detected persistent CHIKV antigens within the synovial fluid of a patient suffering from chronic arthralgia. Moreover, experimental studies in CHIKV infected animal indicate that the joint is the most highly infected tissue, making it plausible that incomplete viral antigen clearance in this anatomical site may account for the long-term symptoms [26]. RRV has been shown to persist in vitro in mouse macrophages, and a model of RRV chronic arthritis suggests that viral persistence may account for chronic disease [40]. RRV and CHIKV have been shown to be weakly tropic for macrophages in vitro $[41,42]$ but the presence of antibodies dramatically increased RRV entry into macrophage [42]. Further studies will be required to assess the role of macrophages, as well the role of persistent infection and antibodies in chronic arthritis caused by CHIKV.

In sum, this study furthers our understanding of the pathophysiology of CHIKV chronic arthralgia, a prerequisite for the development of efficient therapeutic strategies and for assessing the burden of disease inflicted upon populations affected by epidemic Chikungunya disease.

\section{Supporting Information}

Supporting Information S1 Strobe checklist for cohort study. (DOC)

Supporting Information S2 Questionnaire used for the study. (DOCX)

Supporting Information S3 Modelisation and estimation of spatiotemporal dynamics of arthralgia.

(DOC)

Table S1 Likelihood ratio tests testing the complete model against different submodels.

(DOC)

Table S2 Estimation of the annual economic impact of CHIKV long term arthralgia.

(DOG)

\section{Author Contributions}

Conceived and designed the experiments: AM MLA ML. Performed the experiments: AM FS SK. Analyzed the data: CS TC YM FC MLA ML AM. Wrote the paper: CS TC ML AM. 


\section{References}

1. Brighton SW, Prozesky OW, de la Harpe AL (1983) Chikungunya virus infection. A retrospective study of 107 cases. South African medical journal = Suid-Afrikaanse tydskrif vir geneeskunde 63: 313-315.

2. Calabrese LH (2008) Emerging viral infections and arthritis: the role of the rheumatologist. Nature clinical practice Rheumatology 4: 2-3.

3. Dupuis-Maguiraga L, Noret M, Brun S, Le Grand R, Gras G, et al. (2012) Chikungunya disease: infection-associated markers from the acute to the chronic phase of arbovirus-induced arthralgia. PLoS neglected tropical diseases 6: e1446.

4. Enserink M (2006) Infectious diseases. Massive outbreak draws fresh attention to little-known virus. Science (New York, NY) 311: 1085.

5. Gerardin P, Barau G, Michault A, Bintner M, Randrianaivo H, et al. (2008) Multidisciplinary prospective study of mother-to-child chikungunya virus infections on the island of La Reunion. PLoS medicine 5: e60.

6. Lemant J, Boisson V, Winer A, Thibault L, André H, et al. (2008) Serious acute chikungunya virus infection requiring intensive care during the Reunion Island outbreak in 2005-2006. Critical Care Medicine 36: 2536-2541.

7. Schuffenecker I, Iteman I, Michault A, Murri S, Frangeul L, et al. (2006) Genome microevolution of chikungunya viruses causing the Indian Ocean outbreak. PLoS medicine 3: e263.

8. Burt FJ, Rolph MS, Rulli NE, Mahalingam S, Heise MT (2012) Chikungunya: a re-emerging virus. Lancet 379: 662-671.

9. Mavalankar D, Shastri P, Raman P (2007) Chikungunya epidemic in India: a major public-health disaster. The Lancet infectious diseases 7: 306-307.

10. Rezza G, Nicoletti L, Angelini R, Romi R, Finarelli AC, et al. (2007) Infection with chikungunya virus in Italy: an outbreak in a temperate region. Lancet 370 : $1840-1846$.

11. Staikowsky F, Talarmin F, Grivard P, Souab A, Schuffenecker I, et al. (2009) Prospective study of Chikungunya virus acute infection in the Island of La Réunion during the 2005-2006 outbreak. PLoS ONE 4: e7603.

12. Grivard P, Le Roux K, Laurent P, Fianu A, Perrau J, et al. (2007) Molecular and serological diagnosis of Chikungunya virus infection. Pathologie-biologie 55: 490-494.

13. Matheus S, Deparis X, Labeau B, Lelarge J, Morvan J, et al. (2005) Discrimination between primary and secondary dengue virus infection by an immunoglobulin $\mathrm{G}$ avidity test using a single acute-phase serum sample. Journal of clinical microbiology 43: 2793-2797.

14. Julvez J, Michault A, Galtier J, el Amine Ali Halidi M, Vidal JM (1990) [Seroepidemiologic evaluation of malaria in Mayotte (Comoro archipelago) 1984-1988]. Bulletin de la Societe de pathologie exotique 83: 658-668.

15. Bouquillard E, Combe B (2009) A report of 21 cases of rheumatoid arthritis following Chikungunya fever. A mean follow-up of two years. Joint Bone Spine 76: $654-657$.

16. Outhred AC, Kok J, Dwyer DE (2011) Viral arthritides. Expert review of antiinfective therapy 9: 545-554.

17. Borgherini G, Poubeau P, Jossaume A, Gouix A, Cotte L, et al. (2008) Persistent arthralgia associated with chikungunya virus: a study of 88 adult patients on reunion island. Clinical infectious diseases : an official publication of the Infectious Diseases Society of America 47: 469-475.

18. Larrieu S, Pouderoux N, Pistone T, Filleul L, Receveur MC, et al. (2010) Factors associated with persistence of arthralgia among Chikungunya virus-infected travellers: report of 42 French cases. Journal of clinical virology : the official publication of the Pan American Society for Clinical Virology 47: 85-88.

19. Sissoko D, Malvy D, Ezzedine K, Renault P, Moscetti F, et al. (2009) Postepidemic Chikungunya disease on Reunion Island: course of rheumatic manifestations and associated factors over a 15-month period. PLoS neglected tropical diseases 3: e389.

20. Moro ML, Grilli E, Corvetta A, Silvi G, Angelini R, et al. (2012) Long-term chikungunya infection clinical manifestations after an outbreak in Italy: A prognostic cohort study. The Journal of infection 65: 165-72.

21. Couturier E, Guillemin F, Mura M, Léon L, Virion J-M, et al. (2012) Impaired quality of life after chikungunya virus infection: a 2-year follow-up study. Rheumatology 51: 1315-22

22. Chopra A, Anuradha V, Ghorpade R, Saluja M (2012) Acute Chikungunya and persistent musculoskeletal pain following the 2006 Indian epidemic: a 2-year prospective rural community study. Epidemiology and infection 140: 842-850.
23. Mathew AJ, Goyal V, George E, Thekkemuriyil DV, Jayakumar B, et al. (2011) Rheumatic-musculoskeletal pain and disorders in a naïve group of individuals 15 months following a Chikungunya viral epidemic in south India: a population based observational study. International Journal of Clinical Practice 65: 13061312 .

24. Kularatne SAM, Weerasinghe SC, Gihan C, Wickramasinghe S, Dharmarathne S, et al. (2012) Epidemiology, clinical manifestations, and long-term outcomes of a major outbreak of chikungunya in a hamlet in sri lanka, in 2007: a longitudinal cohort study. Journal of Tropical Medicine 2012: 639178.

25. de Andrade DC, Jean S, Clavelou P, Dallel R, Bouhassira D (2010) Chronic pain associated with the Chikungunya Fever: long lasting burden of an acute illness. BMC infectious diseases 10: 31.

26. Couderc T, Chretien F, Schilte C, Disson O, Brigitte M, et al. (2008) A mouse model for Chikungunya: young age and inefficient type-I interferon signaling are risk factors for severe disease. PLoS pathogens 4: e29.

27. Labadie K, Larcher T, Joubert C, Mannioui A, Delache B, et al. (2010) Chikungunya disease in nonhuman primates involves long-term viral persistence in macrophages. The Journal of clinical investigation 120: 894-906.

28. Gardner J, Anraku I, Le TT, Larcher T, Major L, et al. (2010) Chikungunya virus arthritis in adult wild-type mice. Journal of virology 84: 8021-8032.

29. Economopoulou A, Dominguez M, Helynck B, Sissoko D, Wichmann O, et al. (2009) Atypical Chikungunya virus infections: clinical manifestations, mortality and risk factors for severe disease during the 2005-2006 outbreak on Reunion. Epidemiology and infection 137: 534-541.

30. Soumahoro MK, Boelle PY, Gauzere BA, Atsou K, Pelat C, et al. (2011) The Chikungunva epidemic on La Reunion Island in 2005-2006: a cost-of-illness study. PLoS neglected tropical diseases 5: el197.

31. Soumahoro M-K, Gérardin P, Boëlle P-Y, Perrau J, Fianu A, et al. (2009) Impact of Chikungunya virus infection on health status and quality of life: a retrospective cohort study. PLoS ONE 4: e7800.

32. Hoarau JJ, Jaffar Bandjee MC, Krejbich Trotot P, Das T, Li-Pat-Yuen G, et al. (2010) Persistent chronic inflammation and infection by Chikungunya arthritogenic alphavirus in spite of a robust host immune response. Journal of immunology 184: 5914-5927.

33. Chow A, Her Z, Ong EK, Chen JM, Dimatatac F, et al. (2011) Persistent arthralgia induced by Chikungunya virus infection is associated with interleukin6 and granulocyte macrophage colony-stimulating factor. The Journal of infectious diseases 203: 149-157.

34. Werneke SW, Schilte C, Rohatgi A, Monte KJ, Michault A, et al. (2011) ISG15 is critical in the control of Chikungunya virus infection independent of UbE1L mediated conjugation. PLoS pathogens 7: e1002322.

35. Schilte C, Couderc T, Chretien F, Sourisseau M, Gangneux N, et al. (2010) Type I IFN controls chikungunya virus via its action on nonhematopoietic cells. The Journal of experimental medicine 207: 429-442.

36. Her Z, Malleret B, Chan M, Ong EKS, Wong SC, et al. (2010) Active Infection of Human Blood Monocytes by Chikungunya Virus Triggers an Innate Immune Response. The Journal of Immunology 184: 5903-5913.

37. Gerardin P, Fianu A, Michault A, Mussard C, Boussaid K, et al. (2013) Predictors of Chikungunya rheumatism: a prognostic survey ancillary to the TELECHIK cohort study. Arthritis research \& therapy 15: R9.

38. Kam YW, Simarmata D, Chow A, Her Z, Teng TS, et al. (2012) Early appearance of neutralizing immunoglobulin G3 antibodies is associated with chikungunya virus clearance and long-term clinical protection. The Journal of infectious diseases 205: 1147-1154.

39. Laine M, Luukkainen R, Toivanen A (2004) Sindbis viruses and other alphaviruses as cause of human arthritic disease. J Intern Med 256: 457-471.

40. Way SJ, Lidbury BA, Banyer JL (2002) Persistent Ross River virus infection of murine macrophages: an in vitro model for the study of viral relapse and immune modulation during long-term infection. Virology 301: 281-292.

41. Sourisseau M, Schilte C, Casartelli N, Trouillet C, Guivel-Benhassine F, et al. (2007) Characterization of reemerging chikungunya virus. PLoS pathogens 3: e89.

42. Linn ML, Aaskov JG, Suhrbier A (1996) Antibody-dependent enhancement and persistence in macrophages of an arbovirus associated with arthritis. The Journal of general virology 77 (Pt 3): 407-411. 